\title{
RAPID LEFT VENTRICULAR FILLING AND STASIS IN MITRAL REGURGITATION
}

\author{
BY \\ P. G. F. NIXON AND G. H. WOOLER \\ From the Department of Thoracic Surgery and Medicine in the General Infirmary at Leeds
}

Received July 12, 1960

In 1902 Mackenzie described the jugular pulse tracing from a patient with atrial fibrillation (Fig. 1). Pressure declined as the ventricle filled, and rose again before the ventricle contracted. He took this to be a sign that the ventricle filled some time before it contracted, and attributed the rise in venous pressure to stasis. In 1906 Yandell Henderson described the volume changes of the beating mammalian heart and showed that ventricular filling is accomplished rapidly during a phase that begins with the opening of the atrio-ventricular valve and ends with the muscle ceasing to relax. Blood flow into the ventricle is negligible during the remainder of diastole. He gave the name diastasis to the latter period of "rest" or stasis to distinguish it from the former phase of "ventricular relaxation and refilling". Furthermore he confirmed Mackenzie's observation that

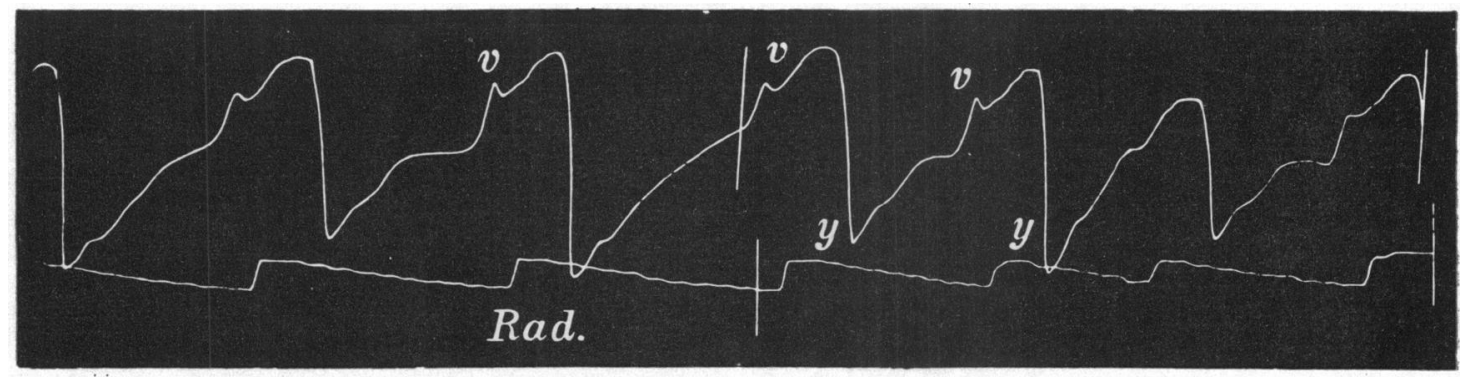

FIG. 1.-Simultaneous tracings of the jugular and radial pulses, showing period of stasis from $y$ to $v$.

alterations in heart rate are effected almost entirely by the lengthening or shortening of the period of stasis. Gibson (1907) and Hirschfelder (1907) described jugular venous stasis waves and third heart sounds in healthy young persons, and the latter referred to the waves as " $h$ " waves.

Wiggers (1921) observed that left atrial pressure in the dog declines during the phase of rapid ventricular filling and may rise again before the next atrial contraction if the heart rate is slow enough. Like Mackenzie he considered such a rise in atrial pressure diagnostic of ventricular stasis. Braunwald et al. (1955) showed the phases of the cardiac cycle in man to be similar to those demonstrated by Wiggers in the experimental animal, and found that the rise in left atrial pressure that identifies ventricular stasis did not occur in patients with mitral stenosis, presumably because obstruction at the mitral orifice prevented rapid ventricular filling. In the same year Owen and Wood observed a gradual decline in left atrial pressure, and inferred slow ventricular filling in patients with mitral stenosis. In their cases of mitral regurgitation the left atrial pressure fell rapidly to ventricular level soon after the mitral valve opened. The subsequent rise 
in left atrial pressure that indicates stasis may be seen in Figures 12 and 13 of Owen and Wood's paper. By percutaneous puncture Fox et al. (1956) obtained direct measurements of left atrial pressure in man and agreed with the conclusions of Owen and Wood. Morrow and his colleagues (1957) recorded direct left atrial pressure tracings by the transbronchial route, and found the ascent characteristic of ventricular stasis occurred in six out of fourteen cases of mitral regurgitation; in seven the heart rate was too rapid for stasis to occur. These authors found no evidence of stasis in the left atrial pressure records from 39 patients with mitral stenosis, although they considered it might be expected to occur in mild cases if left atrial and ventricular pressures could equalize rapidly. In 1958 Ross et al. reported evidence of ventricular stasis in the left atrial pressure tracings from 15 patients with pure mitral regurgitation; in a further eight patients the heart rate was thought to be too rapid for diastasis to exist. On the other hand, Neustadt and Shaffer (1959) rarely saw evidence of ventricular stasis in the left atrial pressure tracings from patients with mitral regurgitation, and Hancock (1959) considered that the diastolic portion of the left atrial pressure pulse did not necessarily distinguish cases of predominant mitral stenosis from cases of predominant mitral regurgitation. Unfortunately neither Neustadt and Shaffer nor Hancock stated whether their tracings were obtained at heart rates slow enough for diastasis to occur.

From these observations it is clear that stasis causes a late diastolic rise in left atrial pressure and signals the achievement of left ventricular filling in the normal heart and in certain patients with mitral regurgitation. Tachycardia abolishes diastasis, and severe mitral stenosis prevents the achievement of ventricular filling.

The diastolic rise in left atrial pressure that marks the period of ventricular stasis and extends from the nadir of the " $y$ " descent to the inscription of the "a" or " $c$ " wave does not appear to have a name. For the sake of brevity it will be called the stasis wave in the remainder of this paper.

The purpose of this communication is to examine the criteria by which left ventricular stasis may be recognized in clinical practice, and to consider the significance of the stasis wave in patients with mitral regurgitation.

\section{Material AND Methods}

Twenty-four patients with atrial fibrillation and the pansystolic murmur of mitral regurgitation were selected for study on the grounds of their freedom from evidence of hypertensive, ischæmic, or aortic valvular disease. Each was severely disabled by his mitral lesion.

At valvotomy or open-heart operation the presence of regurgitation was confirmed, and the long diameter of the mitral valve measured by palpation.

Pre-operative transeptal left heart catheterization (Ross, 1959) was performed in the manner described by Nixon (1960). Left atrial pressure was recorded simultaneously with the pulmonary and mitral phonocardiogram and the electrocardiogram. Care was taken to avoid filtering out the third heart sound and the onset of the mitral diastolic murmur (Fig. 2A). To avoid respiratory distortion of the intra-cardiac pressure pulse, all records were taken during an expiratory pause. Thirty to forty complexes obtained at a paper speed of $80 \mathrm{~mm}$./second were examined in each case. The majority of left atrial pressure tracings were obtained through a Clay-Adams P.E. 50 catheter $90 \mathrm{~cm}$. in length, using a Statham P23 Db transducer with an N.E.P. carrier amplifier and recording camera. High or medium frequency phonocardiograms (Leatham, 1952) were recorded with N.E.P. apparatus.

Two varieties of mitral diastolic murmur occurred-those with a quiet onset and those with a loud onset. A third heart sound was said to be present when vibrations twice the size of the latter variety of murmur occurred shortly after its beginning.

A stasis wave was considered present in the left atrial pulse tracing when pressure rose from the nadir of the " $y$ " descent to the beginning of the " $c$ " wave during an expiratory pause. Entry of the catheter into the left ventricle in patients with mitral regurgitation has shown that the left atrial stasis wave is similar to the left ventricular late diastolic pressure curve (Fig. 2B). In order to determine the shortest period of diastole in which stasis could be demonstrated, measurement was 


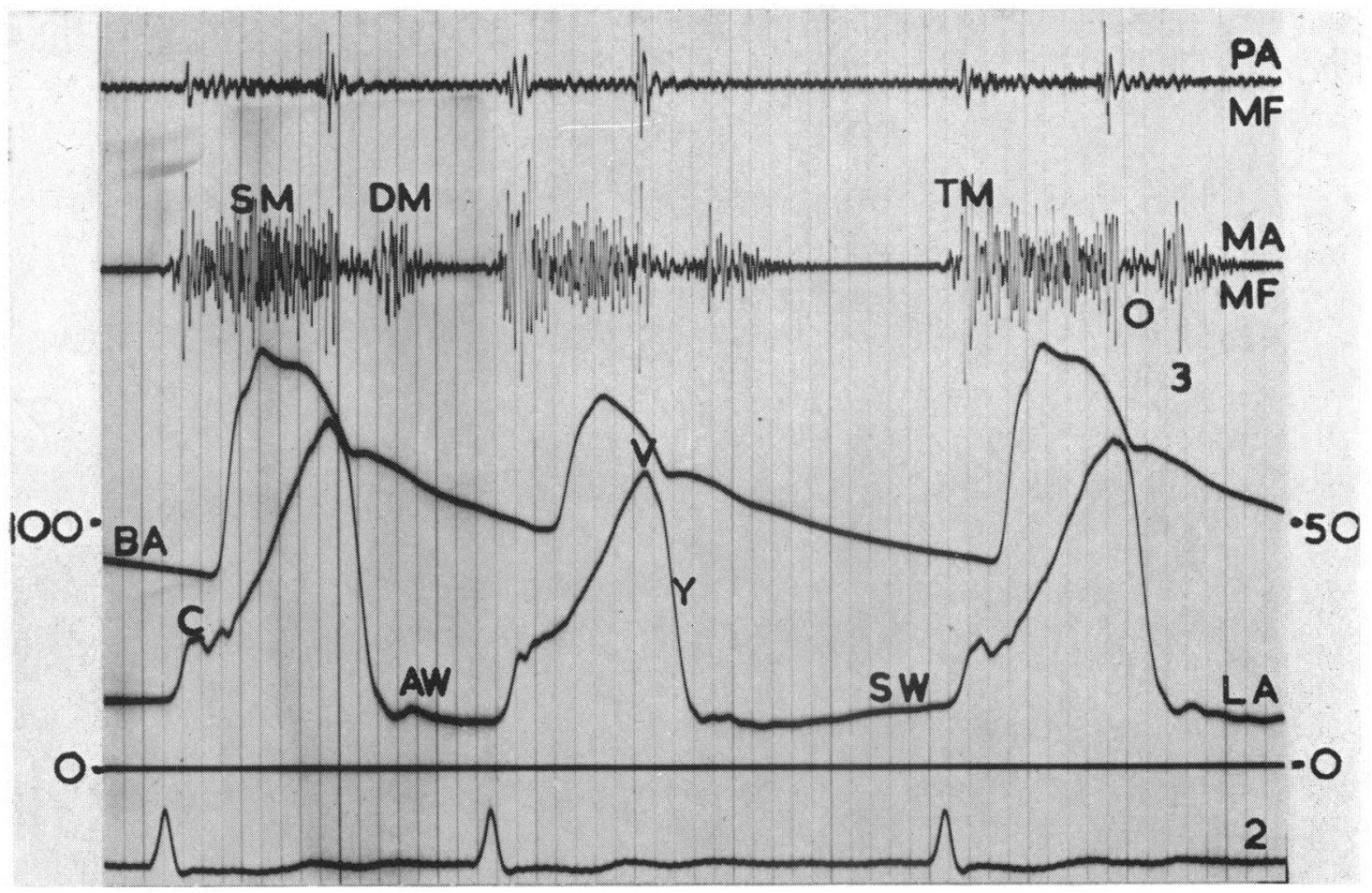

Fig. 2A.-Simultaneous brachial arterial and left atrial pressure pulses, with pulmonary and mitral phonocardiograms and lead II electrocardiogram from a case of severe mitral regurgitation. The scale $(\mathrm{mm}$. $\mathrm{Hg}$, related to the sternal angle) for the brachial pulse is on the left; for the left atrial pulse on the right.

PA = pulmonary area

$M F=$ medium frequency

$\mathrm{BA}=$ brachial arterial pulse

$\mathrm{LA}=$ left atrial pressure pulse

$\mathbf{S M}=$ pansystolic murmur of mitral regurgitation

$\mathrm{DM}=$ mitral diastolic murmur with loud onset

$\mathrm{O}=$ small opening snap vibrations

$3=$ third heart sound of rapid left ventricular filling

$\mathrm{V}$ and $\mathrm{C}=$ left atrial " $\mathrm{v}$ " and "c" waves

$Y=$ left atrial " $y$ " descent

AW=annular ascent wing, Radner (1957)

$\mathrm{SW}=$ stasis wave marking the period of diastasis.

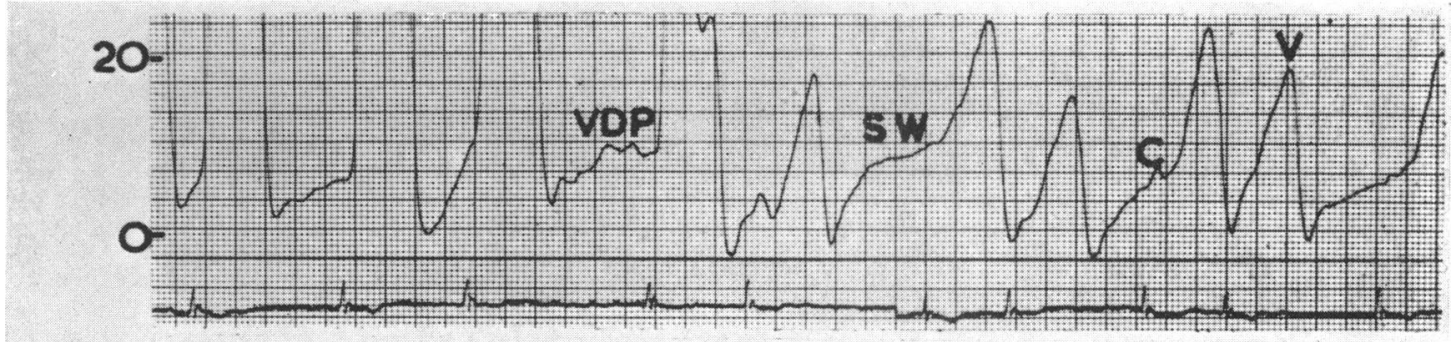

\section{mm.Hg. WITHDRAWAL FROM LEFT VENTRICLE TO ATRIUM}

Fig. 2B.-Withdrawal tracing from left ventricle to left atrium in a case of severe mitral regurgitation. (Zero sternal angle.) The left ventricular systolic peaks are not shown.

VDP = left ventricular late diastolic pressure

$\mathrm{SW}=$ left atrial stasis wave

$V$ and $C=$ left atrial " $v$ " and "c" waves. 
made of the shortest " $v$ " apex to " $c$ " interval in which a stasis wave occurred. In tracings free from stasis waves note was made of the longest " $v$ " apex to " $c$ " intervals present in the tracings.

The average left atrial " $v$ " peak pressure was measured in every case, and related to the sternal angle.

\section{RESULTS}

Figure 2 shows examples of records obtained at left heart catheterization in cases of severe mitral regurgitation. The important findings are tabulated (see p. 167).

It may be seen from the table that in the ten cases with mitral orifices measuring $1.7 \mathrm{~cm}$. or less the mitral diastolic murmur began quietly, and neither a third heart sound nor a stasis wave occurred. In every one of the fourteen patients with mitral orifices measuring $1.8 \mathrm{~cm}$. or more the mitral diastolic murmur began loudly and a third heart sound was recorded; in only one was there failure to demonstrate a stasis wave. The longest " $v$ " apex to "c" interval observed in the first group ranged between 0.64 and 1.6 seconds. In thirteen patients with larger orifices stasis waves occurred in " $v$ " apex to " $c$ " intervals ranging from 0.2 to 1.5 seconds. The magnitude of left atrial pressure at the apex of the " $v$ " wave appeared to bear no consistent relationship to the occurrence of a stasis wave (Fig. 3) or to the brevity of the shortest " $v$ " to " $c$ " interval in which stasis occurred (Fig. 4).

\section{- diastasis present o diastasis absent}

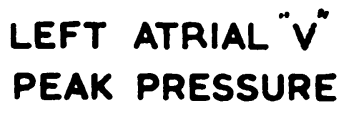

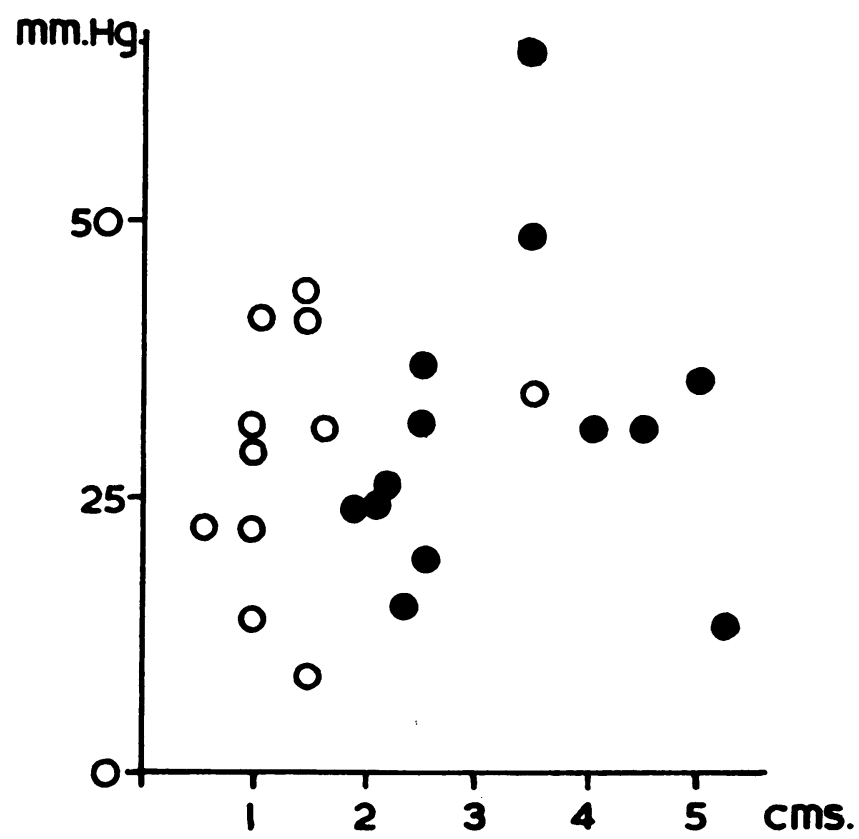

\section{LONG DIAMETER OF THE MITRAL ORIFICE}

FIG. 3.-Left atrial "v" peak pressure plotted against mitral orifice long diameter in 24 patients with mitral regurgitation. A stasis wave was present in all but one of the cases in which the mitral orifice measured $1.8 \mathrm{~cm}$. or more; and in none of those with smaller orifices. There is no apparent relationship between " $v$ " peak pressure and either orifice size or the occurrence of a stasis wave. 


\section{LEFT ATRIAL" $V "$ PEAK PRESSURE}

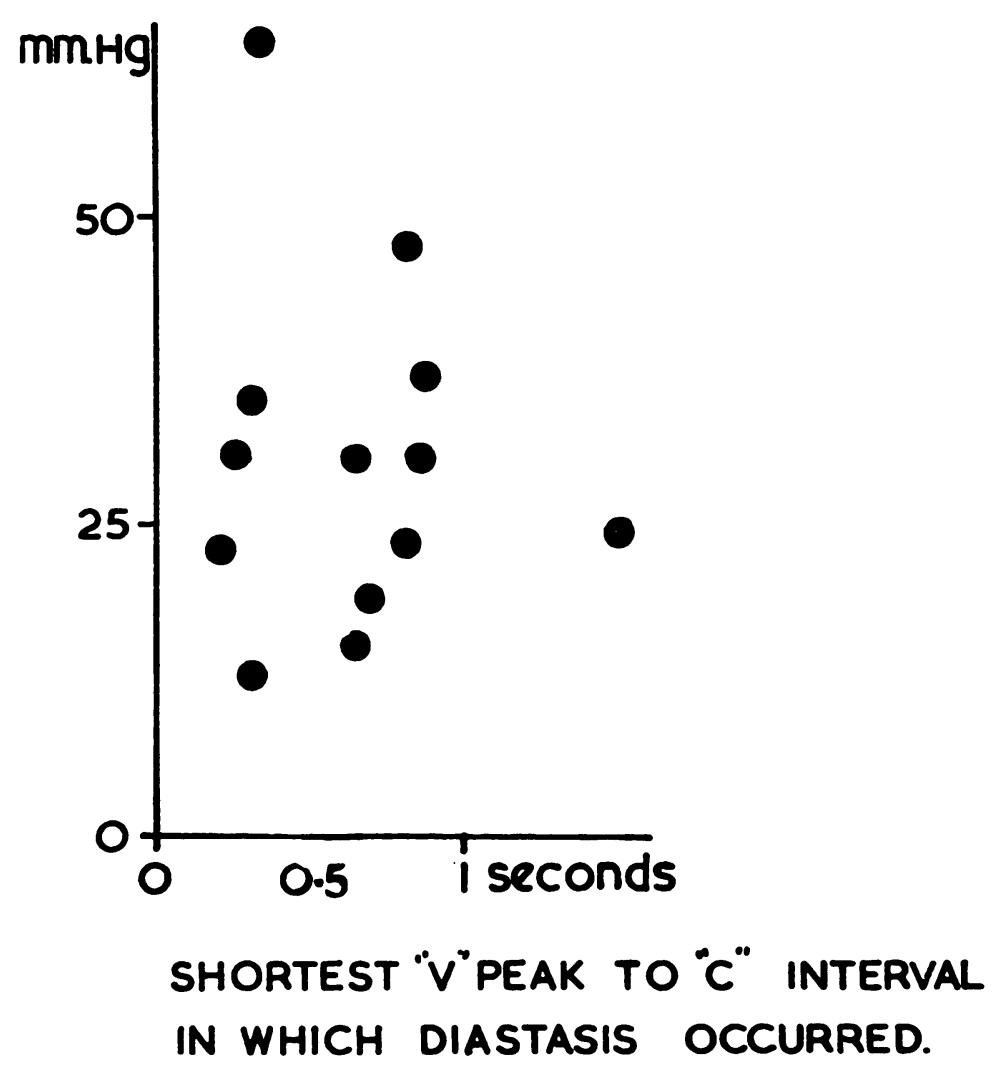

FIG. 4.-Left atrial " $v$ " peak pressure plotted against the shortest " $v$ " peak to " $c$ " interval that contained a stasis wave. There is no apparent relationship between the height of the " $v$ " wave and the length of the interval.

\section{Discussion}

Patients with the pansystolic murmur of mitral regurgitation who are severely disabled by their mitral valve disease may be suffering chiefly from obstruction or from regurgitation. Since it is probably the size of the mitral orifice that determines which physiological derangement is the more severe, its pre-operative assessment may be of greater value than attempts to measure regurgitant flow. It is difficult to define the concepts of "predominant stenosis" and "predominant regurgitation" without first relating them to orifice size.

A loudly beginning diastolic murmur and a frequently occurring third heart sound identify a mitral orifice measuring $2.0 \mathrm{~cm}$. or more in patients disabled from mitral valve disease (Nixon and Wooler, 1960). The present studies suggest that ventricular stasis has similar diagnostic significance. This is not surprising if McKusick (1958) is correct in his view that rapid ventricular filling and an alteration in the volume-elasticity characteristics of the left ventricle are involved in the production of the third heart sound of mitral regurgitation. The stasis wave both signals the completion of a phase of rapid ventricular filling and gives a measurement of the extent to which the left ventricular end-diastolic pressure is raised. Ross et al. (1958) have drawn attention to the great elevation of left ventricular end-diastolic pressure that can occur in mitral regurgitation and their experience has been confirmed in the present series of patients (Fig. 2B).

The stasis wave has been defined as a rise in left atrial pressure from the nadir of the " $y$ " descent to the beginning of the " $a$ " or " $c$ " wave. There is one situation in which it may falsely appear to be present, and one in which it does not mark ventricular stasis. If the left atrial pressure pulse is recorded while breathing continues the respiratory increase in intra-thoracic pressure may give 


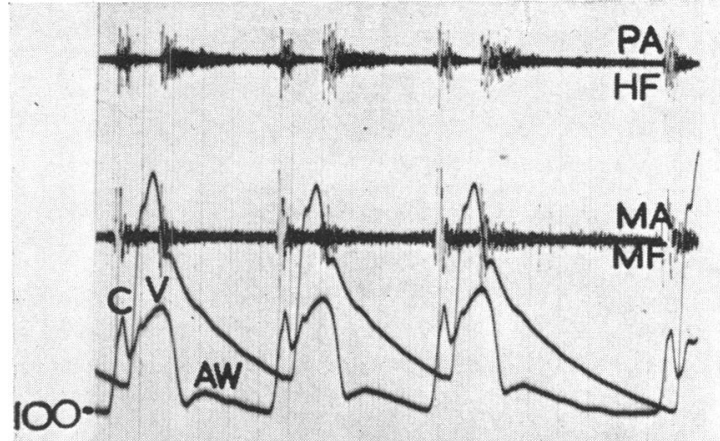

O

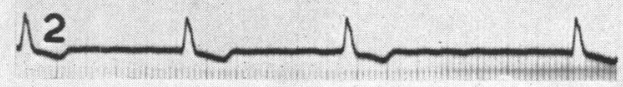

RESPIRATORY PAUSE
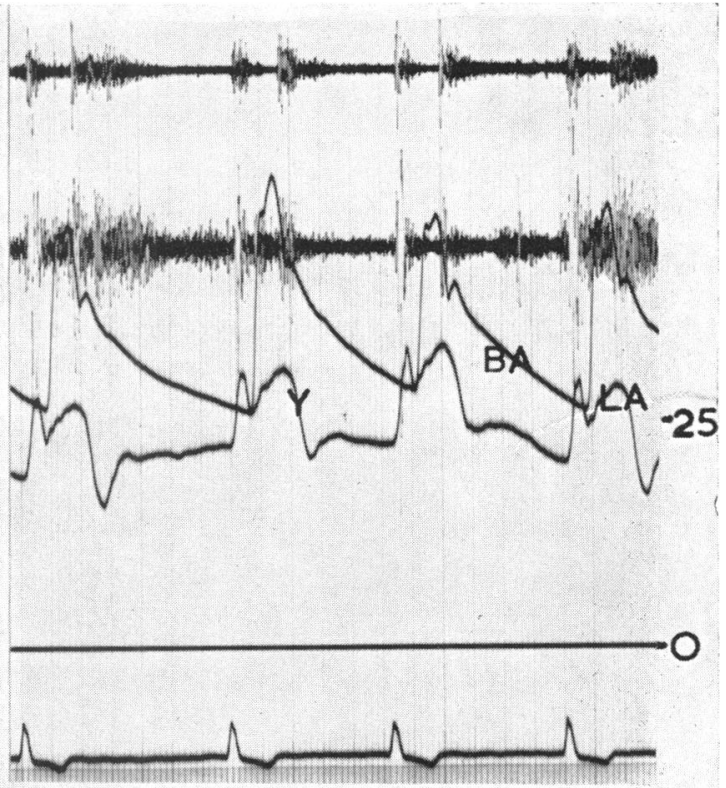

NORMAL BREATHING

FIG. 5.-Simultaneous left atrial and brachial arterial pressure pulses with pulmonary and mitral phonocardiograms and lead II electrocardiogram from a case of severe mitral stenosis. The scale (mm. $\mathrm{Hg}$, related to the sternal angle) for the brachial pulse is on the left; for the left atrial pulse on the right.

The tracing taken in an expiratory pause shows that the " $y$ " descent declines gradually in late diastole. Respiratory distortion of the base line during normal breathing has given the " $y$ " descent the false appearance of a stasis wave in late diastole.

PA= pulmonary area

MA $=$ mitral area

$\mathrm{HF}=$ high frequency

MF =medium frequency

$\mathrm{BA}=$ brachial arterial pressure pulse

$\mathrm{LA}=$ left atrial pressure pulse

$C, V$ and $Y=$ left atrial " $c$ " and " $v$ " waves, and the " $y$ " descent.

AW=annular ascent wing. Radner (1957).

\section{LEFT VENTRICLE}

\section{LEFT ATRIUM}

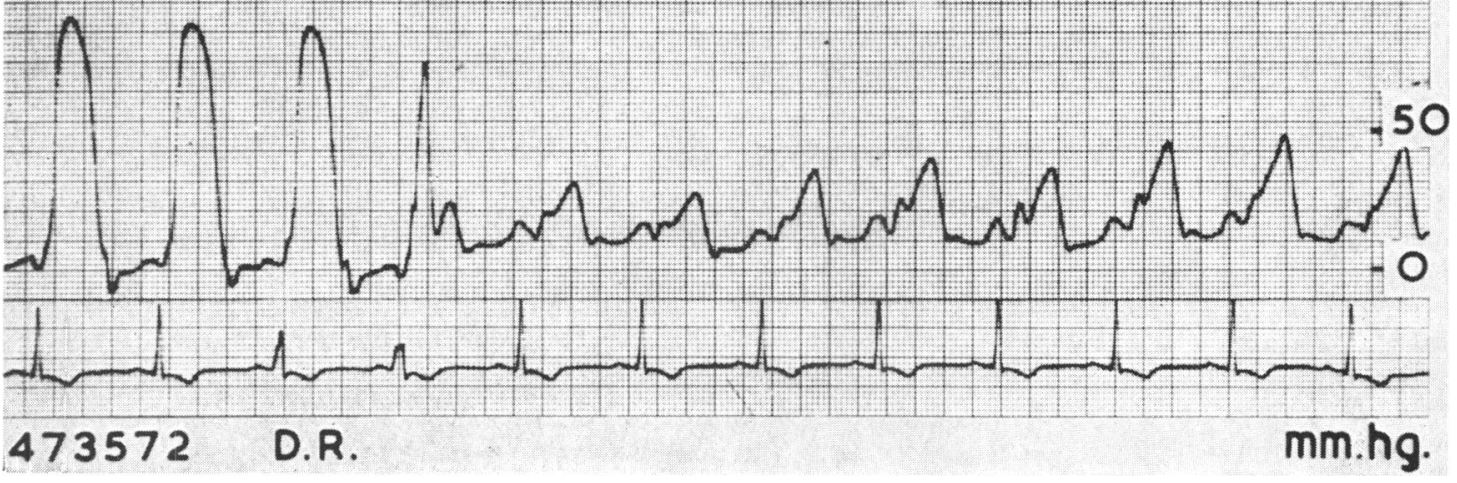

Fig. 6.-Withdrawal tracing from left ventricle to atrium from a case of myxoma of the left atrium. 
TABLE

\begin{tabular}{|c|c|c|c|c|c|c|c|c|}
\hline \multirow[t]{2}{*}{ Patient } & \multirow[t]{2}{*}{$\begin{array}{l}\text { Stasis } \\
\text { wave }\end{array}$} & \multirow{2}{*}{$\begin{array}{c}\text { Mitral } \\
\text { orifice long } \\
\text { diameter } \\
\mathrm{cm} .\end{array}$} & \multirow{2}{*}{$\begin{array}{l}\text { Left atrial } \\
\text { pressure } \\
\text { at "v" } \\
\mathrm{mm} . \mathrm{Hg}\end{array}$} & \multirow{2}{*}{$\begin{array}{l}\text { Shortest "v" } \\
\text { apex to "c" } \\
\text { interval in } \\
\text { which a } \\
\text { stasis wave } \\
\text { occurred } \\
\text { (sec.) }\end{array}$} & \multirow{2}{*}{$\begin{array}{l}\text { Longest "v" } \\
\text { apex to "c" } \\
\text { interval } \\
\text { present in } \\
\text { patients } \\
\text { without stasis } \\
\text { waves (sec.) }\end{array}$} & \multirow{2}{*}{$\begin{array}{l}\text { Third heart } \\
\text { sound of } \\
\text { rapid left } \\
\text { ventricular } \\
\text { filling }\end{array}$} & \multicolumn{2}{|c|}{$\begin{array}{c}\text { Mitral diastolic } \\
\text { murmur }\end{array}$} \\
\hline & & & & & & & $\begin{array}{l}\text { Quiet } \\
\text { onset }\end{array}$ & $\begin{array}{l}\text { Loud } \\
\text { onset }\end{array}$ \\
\hline $\begin{array}{r}1 \\
2 \\
3 \\
4 \\
5 \\
6 \\
7 \\
8 \\
9 \\
10 \\
11 \\
12 \\
13 \\
14 \\
15 \\
16 \\
17 \\
18 \\
19 \\
20 \\
21 \\
22 \\
23 \\
24\end{array}$ & $\begin{array}{l}0 \\
0 \\
0 \\
0 \\
0 \\
0 \\
0 \\
0 \\
0 \\
0 \\
+ \\
+ \\
+ \\
+ \\
+ \\
+ \\
+ \\
+ \\
+ \\
+ \\
+ \\
+\end{array}$ & $\begin{array}{l}0.5 \\
1.0 \\
1.0 \\
1.0 \\
1.0 \\
1.2 \\
1.5 \\
1.5 \\
1.5 \\
1.7 \\
1.8 \\
2.0 \\
2.2 \\
2.3 \\
2.5 \\
2.5 \\
2.5 \\
3.5 \\
3.5 \\
3.5 \\
4.0 \\
4.5 \\
5.0 \\
5.3\end{array}$ & $\begin{array}{r}22 \\
31 \\
29 \\
22 \\
14 \\
42 \\
8 \\
42 \\
44 \\
31 \\
24 \\
24 \\
25 \\
15 \\
37 \\
19 \\
32 \\
65 \\
48 \\
34 \\
32 \\
31 \\
35 \\
13\end{array}$ & $\begin{array}{l}\bar{Z} \\
\bar{Z} \\
\overline{ } \\
\bar{Z} \\
\overline{-} \\
\overline{-} \\
0.2 \\
0.8 \\
1.5 \\
0.64 \\
0.86 \\
0.68 \\
0.26 \\
0.33 \\
0.8 \\
\overline{0} \\
0.84 \\
0.62 \\
0.28 \\
0.34\end{array}$ & $\begin{array}{l}0.72 \\
0.74 \\
0.7 \\
0.98 \\
1.6 \\
0.54 \\
0.64 \\
1.1 \\
0.7 \\
0.74 \\
= \\
= \\
= \\
= \\
= \\
0.33 \\
= \\
= \\
-\end{array}$ & $\begin{array}{l}0 \\
0 \\
0 \\
0 \\
0 \\
0 \\
0 \\
0 \\
0 \\
0 \\
+ \\
+ \\
+ \\
+ \\
+ \\
+ \\
+ \\
+ \\
+ \\
+ \\
+ \\
+ \\
+ \\
+\end{array}$ & $\begin{array}{l}+ \\
+ \\
+ \\
+ \\
+ \\
+ \\
+ \\
+ \\
+ \\
+ \\
+ \\
0 \\
0 \\
0 \\
0 \\
0 \\
0 \\
0 \\
0 \\
0 \\
0 \\
0 \\
0 \\
0 \\
0\end{array}$ & $\begin{array}{l}0 \\
0 \\
0 \\
0 \\
0 \\
0 \\
0 \\
0 \\
0 \\
0 \\
+ \\
+ \\
+ \\
+ \\
+ \\
+ \\
+ \\
+ \\
+ \\
+ \\
+ \\
+ \\
+ \\
+\end{array}$ \\
\hline
\end{tabular}

an upward swing to a gradually declining "y" descent (Fig. 5). When a left atrial tumour is present the normal mitral valve may become obstructed early in diastole and a rise in atrial pressure results from atrial stasis. This condition can be distinguished from ventricular stasis when the stasis wave is shown to lie at a higher level than the left ventricular diastolic pressure (Fig. 6).

In examining left atrial pressure tracings that do not show stasis waves it may be difficult to decide whether their absence is attributable to tachycardia or to a small mitral orifice. In the present series of patients with orifices measuring $1.7 \mathrm{~cm}$. or less no stasis wave occurred during maximum " $v$ " peak to " $c$ " intervals of 0.64 to 1.6 seconds. In seven out of thirteen patients with larger orifices stasis waves were found in intervals of 0.64 seconds or less. Nevertheless in one patient with an orifice of $2.2 \mathrm{~cm}$. an interval of as long as 1.5 seconds was required for a stasis wave to be recorded. One patient with an orifice of $3.5 \mathrm{~cm}$. failed to show a stasis wave. Tachycardia was present, and the longest " $v$ " apex to " $c$ " interval recorded was 0.33 seconds. It is probable that stasis waves would have been seen at a slower heart rate because third heart sounds occurred in this patient.

It is a valid criticism that the patients with a small orifice might have shown stasis waves if longer " $v$ " apex to " $c$ " intervals had occurred during their investigation, but this does not alter the fact that when 30-40 complexes obtained under similar conditions were examined, stasis waves appeared in 13 out of 14 patients with larger orifices and third heart sounds, and failed to appear in the 10 patients with smaller orifices and mitral diastolic murmurs that began quietly.

A major indication for left heart catheterization in mitral regurgitation will be removed if further experience supports the view that phonocardiography can separate patients with small orifices and predominant obstruction from those with larger orifices, rapid ventricular filling, and predominant regurgitation. Nevertheless' the height of the stasis wave may prove to be a useful 
guide to the severity of left heart disorder in mitral regurgitation, particularly when the results of surgical treatment are assessed.

\section{SUMMARY}

Left ventricular stasis marks the completion of a phase of rapid ventricular filling and is recognized by a rise in left atrial pressure from the nadir of the " $y$ " descent to the inscription of the " $c$ " or " $a$ " wave. It is suggested that this rise in pressure be named the left atrial stasis wave. In a series of patients with mitral regurgitation a close association was found between the occurrence of a stasis wave and the presence of a third heart sound. Stasis waves were found in the left atrial pressure pulse tracings from 13 out of 14 patients with third heart sounds. In each case the mitral orifice long diameter measured $1.8 \mathrm{~cm}$. or more. Neither stasis waves nor third heart sounds were present in the patients with smaller orifices.

The diagnosis of the stasis wave in clinical practice is discussed.

The authors are grateful to Mr. R. Addyman and Staff-Nurse E. M. Kirkbride for zealous technical assistance; and to Mr. J. A. Aylwin for the opportunity to investigate his successfully treated case of left atrial myxoma.

The work has been made possible by generous grants from the Nuffield Foundation, the Endowment Fund of the General Infirmary at Leeds and private benefactors.

\section{REFERENCES}

Braunwald, E., Moscovitz, H. L., Amram, S. S., Lasser, R. P., Sapin, S. O., Himmelstein, A., Ravitch, M. M., and Gordon, A. J. (1955). Circulation, 12, 69.

Fox, I. J., Coolidge, S. W., Connolly, D. C., and Wood, E. H. (1956). Proc. Staff Meet. Mayo Clin., 31, 126.

Hirschfelder, A. D. (1907) Bull. Johns Hopk. Hosp., 18, 265.

Hancock, E. W. (1959). Brit. Heart J., 21, 389.

Henderson, Y. (1906). Amer. J. Physiol., 16, 325.

Gibson, A. G. (1907). Lancet, $2,1380$.

Leatham, A. (1952). Brit. med. Bull., 8, 334.

Mackenzie, J. (1902). The Study of the Pulse. Young, J. Pentland, Edinburgh and London.

McKusick, V. A. (1958). Cardiovascular Sound. The Williams \& Wilkins Company, Baltimore.

Morrow, A. G., Braunwald, E., Haller, A. J., and Sharp, E. (1957). Circulation, 16, 399.

Neustadt, J. E., and Shaffer, A. B. (1959). Amer. Heart J., 58, 675.

Nixon, P. G. F. (1960). Thorax, 15, 225.

, and Wooler, G. H. (1960). Brit. med. J., 2, 1122.

Owen, S. G., and Wood, P. (1955). Brit. Heart J., 17, 41.

Radner, S. (1957). Acta med. Scand., 159, 219.

Ross, J., Jr., Braunwald, E., and Morrow, A. G. (1958). Amer. J. Cardiol., 1, 11.

- (1959). Ann. Surg., 149, 395.

Wiggers, C. J. (1921). Amer. J. Physiol., 56, 415 and 439. 